\title{
Detection of Abnormalities in a Biological Tissue by Diffuse Optical Tomography: A Computational Study
}

\author{
H. Trabelsi, M. Gantri and E. Sediki \\ University of Tunis El Manar, Faculté des Sciences de Tunis \\ Unité de Rayonnement, Département de Physique, Tunis
}

Tunisia

\section{Introduction}

Diffuse optical tomography (DOT) has a real potential as a new medical diagnostic tool. This fact has stimulated considerable research interest in the last years. When it is possible, this technique has some advantages not available in classical medical imaging methods. Among the benefits of this technique, nonionizing radiation, low-cost instrumentation, mobility and possible functional imaging are the most important. Also, DOT could be used in combination with other imaging methods to provide high-resolution structural information. The transparency of tissues is related to absorption and scattering of light and it reaches its maximum in the near-infrared. Interaction of radiation from this range with biological tissues is widely studied [1-5]. In particular, some works was interested to detection of tumor-like inclusions within a biological tissue [6-8].

Most of models of light interaction with biological matter are based on radiative transfer theory [9-13]. The most used parameters in modelling Laser radiation interaction with biological tissue is absorption and scattering. However some other studies evoked a significant variation of refractive index of abnormal biological tissues especially in the near infrared range. More precisely, experimental results [14-17] showed that the tissue of malignant tumors could manifest an increase of the refractive index which could attain until $10 \%$ of that of a normal tissue which encircles them. So, medical imaging by diffuse optical tomography should take advantage from the emergence of a third contrast parameter which is the refractive index. This drove to the appearance of a big number of numerical and fundamental works in the field of radiative transfer in a varying refractive index medium. Among them some papers are interested to varying refractive index biological tissues [18-22].

In this chapter, infrared laser radiation interaction with a biological tissue including a spatially variation of refractive index is studied through a time-dependent radiative transfer model. Our general concern is to contribute at the usability of the radiative transfer theory in a potential optical tomography setting in medical imaging. At this level, studying the effect of refractive index on the transmitted light through a biological rectangular layer is crucial. This should be a contribution to obtaining information about 
abnormal tissue in a typical DOT scheme. However, it is important to note that in a varying refractive index medium, the rays are not straight lines but curves. So even in a rectangular geometry, the varying refractive index radiative transfer equation displays angular derivative terms [23-25]. In this paper, these terms are treated with finite differencing scheme. More precisely, we present a computational model that could be suitable for basic optical tomography forward problem with spatially varying refractive index. We investigate cases concerning optical tomography applications. We consider a biological tissue-like domain submitted to a near infrared light source. Transmitted intensity detected on the boundary is computed. The effect of the embedded nonhomogenous objects on the transmitted signal is studied. Particularly, a possible detection of these objects is discussed. Also, the model is used to study infrared radiation in a multilayered heterogeneous medium. The effect of optical parameters on the transmitted signal is presented. In particular, the effect of refractive index variation is pointed out.

\section{Physical model and numerical implementation}

In this work, the radiative transfer in a human biological tissue is described by using a varying refractive index RTE [26, 27]

$$
\begin{aligned}
& \frac{n(\vec{r})}{c} \frac{\partial I(\vec{r}, \Omega, t)}{\partial t}+\vec{\Omega} . \nabla I(\vec{r}, \vec{\Omega}, t)+\left(\mu_{a}(\vec{r})+\mu_{s}(\vec{r})\right) I(\vec{r}, \Omega, t)+\frac{1}{n(\vec{r})} \nabla n \cdot \nabla_{\vec{\Omega}} I(\vec{r}, \vec{\Omega}, t) \\
& -\frac{2}{n(\vec{r})}(\vec{\Omega} . \nabla n) I(\vec{r}, \vec{\Omega}, t)=S(\vec{r}, \vec{\Omega}, t)+\mu_{s}(r) \int_{0}^{2 \pi} p\left(\vec{\Omega}, \vec{\Omega}^{\prime}\right) I\left(\vec{r}, \vec{\Omega}^{\prime}, t\right) d \vec{\Omega}^{\prime}
\end{aligned}
$$

where $I(\vec{r}, \vec{\Omega}, t)$ is the directional energetic radiance at the spatial position $\vec{r}$ and $n(\vec{r})$ is the refractive index distribution. Equation (1) takes into account the fact that the rays are not straight lines but curves. It involves terms that illustrate the expansion or the contraction of the cross section of the tube of light rays in the medium. On the boundary, the radiance is the sum of the external source contribution and the partly-reflected radiance due to the refractive index mismatch at the boundary

$$
I(\vec{r}, \vec{\Omega}, t)=S\left(\vec{r}_{b}, \vec{\Omega}, t\right)+R I\left(\vec{r}, \vec{\Omega}_{r e f}, t\right), \vec{n}_{b} \cdot \vec{\Omega}<0 \text { and } \vec{n}_{b} \cdot \vec{\Omega}_{r e f}=-\vec{n}_{b} \cdot \vec{\Omega},
$$

where $\vec{r}_{b}$ is a position on the boundary and $\vec{n}_{b}$ is an outer normal unit vector. The reflectivity $R$ can be calculated for each direction using Fresnel's relations. For a twodimensional problem and in Cartesian coordinate system of the $x-y$ plane, the terms due to the refractive index variation can be expressed as

$$
\frac{1}{n} \nabla n . \nabla_{\vec{\Omega}} I-\frac{2}{n}(\vec{\Omega} . \nabla n) I=-\frac{1}{n} \frac{\partial n}{\partial x}\left(\sin \varphi \frac{\partial I}{\partial \varphi}+2 \cos \varphi I\right)+\frac{1}{n} \frac{\partial n}{\partial y}\left(\cos \varphi \frac{\partial I}{\partial \varphi}-2 \sin \varphi I\right),
$$

where $\cos \varphi$ and $\sin \varphi$ are the Cartesian coordinates of the unit direction vector in the $x-y$ plane. In fact we assume that the radiance of out of plane directions is negligible. By using notations $\xi=\cos \varphi$ and $\eta=\sin \varphi$, equation (2) displays the classical form of the angular redistribution term commonly appearing when dealing with spherical and cylindrical geometries with uniform refractive index $[28,29]$ 


$$
\frac{1}{n} \nabla n \cdot \nabla_{\vec{\Omega}} I-\frac{2}{n}(\vec{\Omega} . \nabla n) I=\frac{1}{2 n^{2}}\left\{\frac{\partial n^{2}}{\partial x} \frac{\partial}{\partial \xi}\left[\left(1-\xi^{2}\right) I\right]+\frac{\partial n^{2}}{\partial y} \frac{\partial}{\partial \eta}\left[\left(1-\eta^{2}\right) I\right]\right\}
$$

The angular redistribution terms will be noted

$$
D_{x}=\frac{\partial}{\partial \xi}\left[\left(1-\xi^{2}\right) I\right] \text { and } D_{y}=\frac{\partial}{\partial \eta}\left[\left(1-\eta^{2}\right) I\right]
$$

so equation (3) becomes

$$
\frac{1}{n} \nabla n \cdot \nabla_{\vec{\Omega}} I-\frac{2}{n}(\vec{\Omega} \cdot \nabla n) I=\frac{1}{2 n^{2}}\left\{\frac{\partial n^{2}}{\partial x} D_{x}+\frac{\partial n^{2}}{\partial y} D_{y}\right\}
$$

In our numerical implementation, we use a rectangular domain which is divided into a set of I $x$ J elementary uniform volumes $\Delta \mathrm{V}$ with a uniform unitary depth. The angular discretization is obtained through a discrete ordinate technique. This yields a set of $\mathrm{M}$ discrete directions, $\left\{\varphi_{m}, m=1 . . M\right\}$ giving a set of angular discrete direction cosines $\left\{\left(\xi_{m}, \eta_{m}\right), m=1, \ldots M\right\}$. An orientation depending on the incident ray direction is adopted for each cell [12]. Calculations are done by using integration of equation (1) over an elementary volume $\Delta \mathrm{V}$ for each discrete direction. This gives

$$
\begin{aligned}
& \frac{n}{c} \frac{\partial I_{m, P}}{\partial t}+\Delta y \xi_{m}\left(I_{m, E}-I_{m, W}\right)+\Delta x \eta_{m}\left(I_{m, N}-I_{m, S}\right)+\Delta x \Delta y\left(\mu_{a}+\mu_{s}\right) I_{m, p} \\
& +\frac{1}{n} \frac{\partial n}{\partial x} D_{x, m}+\frac{1}{n} \frac{\partial n}{\partial y} D_{y, m}=\Delta x \Delta y\left(S_{m, P}+\mu_{s} w_{m} p_{m m} I_{m, p}\right. \\
& \left.+\mu_{s} \sum_{m^{\prime}=1, m^{\prime} \neq m}^{M} w_{m} p_{m m^{\prime}} I_{m^{\prime}, p}\right)
\end{aligned}
$$

where $D_{x, m}$ and $D_{y, m}$ are the discrete angular derivative terms at the angular ordinate $\xi_{m}$ and $\eta_{m}$ respectively and $w_{m}$ is a weighting factor. The discrete term of Henyey-Greenstein phase function is written as

$$
p_{m m^{\prime}}=\frac{1-g^{2}}{4 \pi\left(1+g^{2}-2 g\left(\xi_{m} \xi_{m^{\prime}}+\eta_{m} \eta_{m^{\prime}}\right)\right)^{3 / 2}}
$$

If the direction cosines are positive, the directional radiances are known on the faces $\mathrm{W}$ and $\mathrm{S}$ and they are unknown on the faces $\mathrm{E}$ and $\mathrm{N}$ of the $(\mathrm{i}, \mathrm{j})$-cell and also in the centre $\mathrm{P}$. Therefore, we need two complementary relations to eliminate $\Psi_{m, N}$ and $\Psi_{m, E}$, this can be obtained by using interpolation formula

$$
\left\{\begin{array}{l}
I_{m, P}=\alpha I_{m, E}+(1-\alpha) I_{m, W} \\
I_{m, P}=\alpha I_{m, N}+(1-\alpha) I_{m, S}
\end{array}\right.
$$

where $\alpha$ is an interpolation parameter. Using these relations, equation (3) becomes 


$$
\begin{aligned}
& \frac{n}{c} \frac{\partial I_{m, P}}{\partial t}+\frac{\Delta y \xi_{m}}{\alpha}\left(I_{m, P}-I_{m, W}\right)+\frac{\Delta x \eta_{m}}{\alpha}\left(I_{m, P}-I_{m, S}\right)+\Delta x \Delta y\left(\mu_{a}+\mu_{s}\right) I_{m, p} \\
& +\frac{1}{2 n^{2}}\left\{\frac{\partial n^{2}}{\partial x} D_{x}+\frac{\partial n^{2}}{\partial y} D_{y}\right\}=\Delta x \Delta y\left(S_{m, P}+\mu_{s} w_{m} p_{m m} I_{m, p}\right. \\
& \left.+\mu_{s} \sum_{m^{\prime}=1, m^{\prime} \neq m}^{M} w_{m} p_{m m^{\prime}} I_{m^{\prime}, p}\right)
\end{aligned}
$$

For time discretization, an implicit three level second order time differencing scheme is used:

$$
\frac{\partial I_{m, P}}{\partial t}=\frac{3 I_{m, P}^{n+1}-4 I_{m, P}^{n}+I_{m, P}^{n-1}}{2 \Delta t} ; \quad n=1, \ldots n_{\max }
$$

where $\Delta t$ being the discrete time step.

Theoretically, if we know the solution in the $(i, j)$-cell, we can do calculus over the cells $(i+1, j)$ and $(i, j+1)$ using the boundary conditions and the following relations

$$
\left\{\begin{array}{l}
I_{m, w}(i+1, j)=I_{m, E}(i, j) ; i=1, \ldots I-1 \\
I_{m, S}(i, j+1)=I_{m, N}(i, j) ; i=1, \ldots J-1
\end{array}\right.
$$

If the direction cosines are both positive, we get the following equation

$$
\begin{aligned}
& \frac{n}{c} \frac{3 I_{m, i, j}^{n+1}-4 I_{m, i, j}^{n}+I_{m, i, j}^{n-1}}{2 \Delta t}+\frac{\Delta y \xi_{m}}{\alpha}\left(I_{m, i, j}^{n}-I_{m, i-1, j}^{n}\right)+\frac{\Delta x \eta_{m}}{\alpha}\left(I_{m, i, j}^{n}-I_{m, i, j-1}^{n}\right)+ \\
& \Delta x \Delta y\left(\mu_{a}+\mu_{s}\right) I_{m, i, j}^{n}+\frac{1}{2 n^{2}}\left\{\frac{\partial n^{2}}{\partial x} D_{x}+\frac{\partial n^{2}}{\partial y} D_{y}\right\} \\
& =\Delta x \Delta y\left(S_{m, i, j}+\mu_{s} w_{m} p_{m m} I_{m, i, j}^{n}+\mu_{s} \sum_{m^{\prime}=1, m^{\prime} \neq m}^{M} w_{m} p_{m m^{\prime}} I_{m^{\prime}, i, j}^{n}\right)
\end{aligned}
$$

\section{Numerical treatment of angular derivative terms}

The numerical treatment of the angular redistribution terms can be done by using the classical angular differencing scheme $[23,28]$.

$$
\begin{aligned}
D_{x, m, i, j} & =\frac{A_{m+1 / 2} I_{m+1 / 2, i, j}-A_{m-1 / 2} I_{m-1 / 2, i, j}}{w_{m}} . \\
D_{y, m, i, j} & =\frac{B_{m+1 / 2} I_{m+1 / 2, i, j}-B_{m-1 / 2} I_{m-1 / 2, i, j}}{w_{m}}
\end{aligned}
$$

where

$$
I_{m+1 / 2, i, j}=\frac{1}{2}\left(I_{m+1, i, j}+I_{m, i, j}\right) \text { and }
$$




$$
I_{m-1 / 2, i, j}^{k}=\frac{1}{2}\left(I_{m-1, i, j}^{k}+I_{m, i, j}^{k}\right) \text { with } I_{M+1, i, j}=I_{1, i, j}, I_{0, i, j}=I_{M, i, j} .
$$

The coefficients $\mathrm{A}$ and $\mathrm{B}$ are given through the recurrent relations

$$
\begin{gathered}
A_{m+1 / 2}-A_{m+1 / 2}=-2 w_{m} \xi_{m}, \\
B_{m+1 / 2}-B_{m+1 / 2}=-2 w_{m} \eta_{m} \text { with } A_{1 / 2}=B_{1 / 2}=0,
\end{gathered}
$$

and $w_{m}$ being a quadrature weight factor. In this paper, we use a constant weight quadrature and an equally spaced distribution of angular ordinates $(\xi, \eta)$. To solve the above equation we use successive iterations in order to actualise the implicit internal source term in the right member. So, this gives

$$
\begin{aligned}
& \left(I_{m, i, j}^{n+1}\right)^{k+1}=\left[\frac{3 n_{i, j}}{2 c \Delta t}+\frac{\Delta y \xi_{m}}{\alpha}+\frac{\Delta x \eta_{m}}{\alpha}+\Delta x \Delta y\left(\mu_{a, i, j}+\mu_{s, i, j}\right)\right]^{-1} \times \\
& {\left[\begin{array}{l}
\frac{2 n_{i, j}}{c \Delta t} I_{m, i, j}^{n}-\frac{n_{i, j}}{2 c \Delta t} \overline{I_{m, i, j}^{n-1}}+\frac{\Delta y \xi_{m}}{\alpha}\left(I_{m, i-1, j}^{n+1}\right)^{k+1}+\frac{\Delta x \eta_{m}}{\alpha}\left(I_{m, i, j-1}^{n+1}\right)^{k+1} \\
+\frac{1}{2 n_{i, j}^{2}}\left(\Delta y\left(n_{i, j}^{2}-n_{i-1, j}^{2}\right) D_{x, m, i, j}^{k}+\Delta x\left(n_{i, j}^{2}-n_{i, j-1}^{2}\right) D_{y, m, i, j}^{k}\right) \\
+\Delta x \Delta y\left(S_{m, i, j}+\mu_{s} w_{m} p_{m m}\left(I_{m, i, j-1}^{n+1}\right)^{k}+\mu_{s, i, j} \sum_{m^{\prime}=1, m^{\prime} \neq m}^{M} w_{m} p_{m m^{\prime}}\left(I_{m^{\prime}, i, j-1}^{n+1}\right)^{k}\right.
\end{array}\right]}
\end{aligned}
$$

The iteration process is repeated until a convergence criterion is attempted. To improve convergence speed, we use a successive over relaxation method. So the updated value $\left(I_{m, i, j}^{n}\right)^{k+1}$ is a linear combination of the iterated value $\left(I_{m, i, j}^{n}\right)^{k}$ and the previously computed value:

$$
\left(I_{m, i, j}^{n}\right)_{\text {updated }}^{k+1}=\rho\left(I_{m, i, j}^{n}\right)^{k}+(1-\rho)\left(I_{m, i, j}^{n}\right)^{k+1}
$$

where $\rho$ is a relaxation parameter whose value is usually between 1 and 2 . The solution is obtained when the relative discrepancy value:

$$
\varepsilon=\left|\frac{\left(I_{m, i, j}^{n}\right)^{k+1}-\left(I_{m, i, j}^{n}\right)^{k}}{\left(I_{m, i, j}^{n}\right)^{k}}\right|
$$

is smaller than a tolerance value. In that case the result is noted $\bar{I}_{m, i, j}$. In all our calculus, we have taken $10^{-8}$ as a tolerance value. As initial condition, we take a field of null radiances. Also, all our calculations are done in the case of interpolation diamond scheme $(a=0.5)$.

If the direction cosines are not both positive, the precedent equations are valid provided that the orientation WESN of cells is done according to the direction of propagation. In all our 
investigations, the injected power source is assumed to be equivalent to a forward collimated monochromatic intensity placed at a source point on the middle of the bottom side of the boundary. Results shown below are obtained by using a near infrared collimated source with a uniform equivalent intensity value of $50 \mathrm{~mW} . \mathrm{cm}^{-1}$. To present our results, we use the detected fluence rate which is given in a $\left(i_{d}, j d\right)$-detector point on the boundary as

$$
\begin{gathered}
\Phi_{d}=\sum_{m=1}^{M}\left(1-R_{m, i_{d}, j_{d}}\right) w_{m} \bar{I}_{m, i_{d}, j_{d}}, \text { with } \\
R_{m, i_{d}, j_{d}}=\left\{\begin{array}{l}
1 \text { if } \varphi_{m} \succ \arcsin \left(\frac{n_{\text {air }}}{n_{i_{d}, j_{d}}}\right) \\
\frac{1}{2}\left[\left(\frac{n_{i_{d}, j_{d}} \cos \varphi_{m}-n_{\text {air }} \cos \varphi_{t}}{n_{i_{d}, j_{d}} \cos \varphi_{m}+n_{\text {air }} \cos \varphi_{t}}\right)^{2}+\left(\frac{n_{i_{d}, j_{d}} \cos \varphi_{t}-n_{\text {air }} \cos \varphi_{m}}{n_{i_{d}, j_{d}} \cos \varphi_{t}+n_{\text {air }} \cos \varphi_{m}}\right)^{2}\right], \text { else. }
\end{array}\right.
\end{gathered}
$$

where

$$
\varphi_{t}=\arcsin \left(\frac{n_{\text {air }} \sin \varphi_{m}}{n_{i_{d}, j_{d}}}\right) \text { and } n_{\text {air }} \approx 1 .
$$

Also, we make use of a normalized detected fluence rate defined as

$$
\Phi_{N}=\frac{\Phi_{d}}{1 / D \sum_{d=1}^{D} w_{d}{ }_{d} \Phi_{d}},
$$

where $D$ is the number of the detector points on one side of the boundary and $\mathrm{w}_{\mathrm{d}}{ }_{\mathrm{d}}$ is a weighting factor from the generalized trapezoidal integration rule. In all calculations, we have used 28 detector points on each side. Also, all calculus is carried out by using 16 uniformly distributed discrete directions and a space grid of $121 \times 121$ cells.

\section{Results and discussion}

\subsection{First test: A continuous varying refractive index medium}

As a first investigation, we study near infrared radiation transport in a rectangular medium exposed to a continuous collimated source which is placed on the bottom side of the boundary. Figure 1 shows the considered medium, it is assumed to be $3 \mathrm{~cm} \times 3 \mathrm{~cm}$ sized with varying refractive index. Within the medium, we consider a y-axis linear varying refractive index between the extreme values 1.33 and 1.58. It is an increasing- decreasing variation of refractive index RI. To show the effect of the refractive index on detected fluence rate, we have used a weakly absorbing and forward scattering background medium whose optical parameters are shown in figure1. Figure 2 shows the medium response detected on the right side of the boundary in the case of linear and parabolic variation respectively. The detected fluence rate curve presents distinguish increasing and decreasing regions with a peak of transmission when refractive index is close to the minimum value 1.33. Transmission on the boundary is relatively augmented when the refractive index is the closest to the air index. 


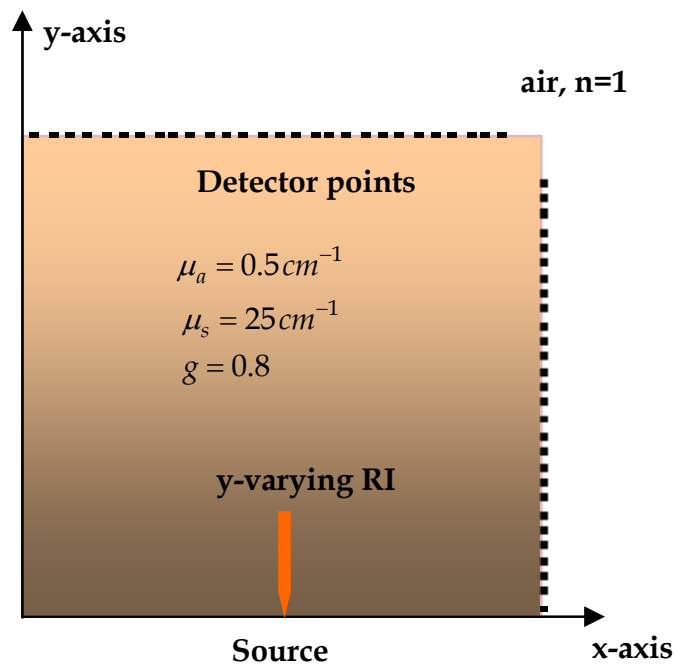

Fig. 1. A test-medium with background properties and detector points
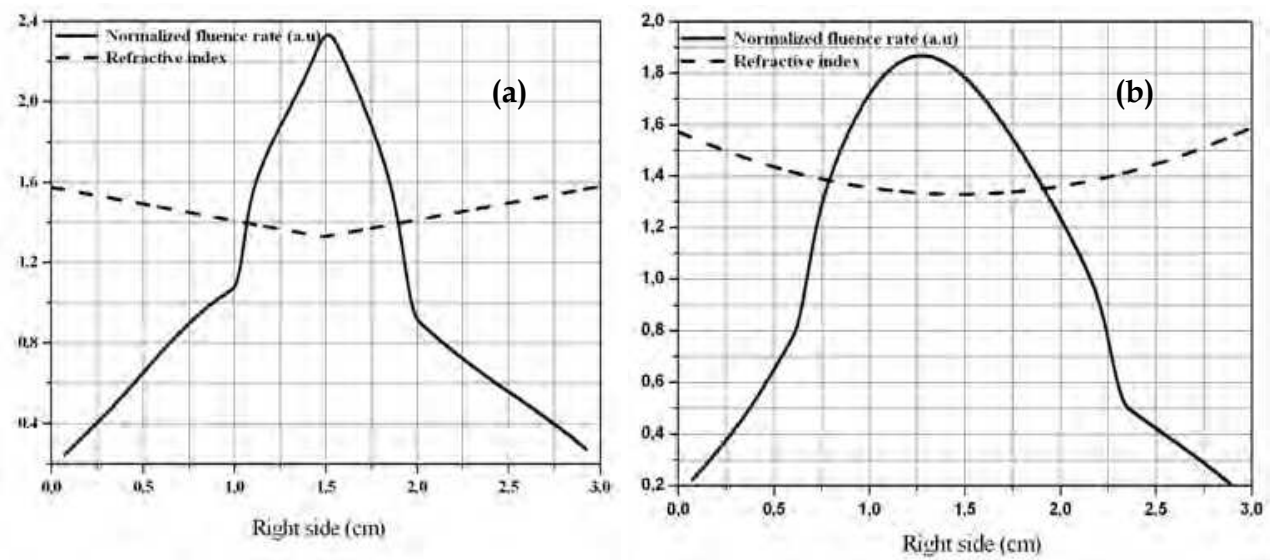

Fig. 2. Response of a continuous varying refractive index medium (a) in the case of a linear variation and (b) in the case of a parabolic variation.

\subsection{Second investigation: Detection of heterogeneous object}

In this investigation, we consider a $3 \mathrm{~cm} \times 3 \mathrm{~cm}$ sized background medium containing a circular heterogeneous object. The area of the object is $0.5 \mathrm{~cm}^{2}$. We limit the analysis to the effect of heterogeneity by increase of the refractive index only. The background refractive index is taken 1.33. The other optical properties are the same as in the precedent investigation for the background and the object. The geometry of the medium and the position of the heterogeneous object are shown in figure 3 . The detected signal on different 
sides of the medium is shown for an increase of refractive index by $5 \%$ and $10 \%$ respectively. There is no significant effect of the object presence on the detected signal on the left side because it is far from the detectors on that side. However there is a visible effect of the object on the right side and on the top side where the detectors are relatively near by the object. In such cases, there is an obvious effect of the heterogeneity on the detected signal especially when the refractive index is increased by $10 \%$. The response shows a distinct distortion of the fluence rate curve. It is occurring on the targets where the object centre is laying exactly. This distortion allows a principled decision on the existence of such object in the medium. These findings highlight the potential of refractive index as a possible detection parameter of a tumour in a surrounding safe tissue.
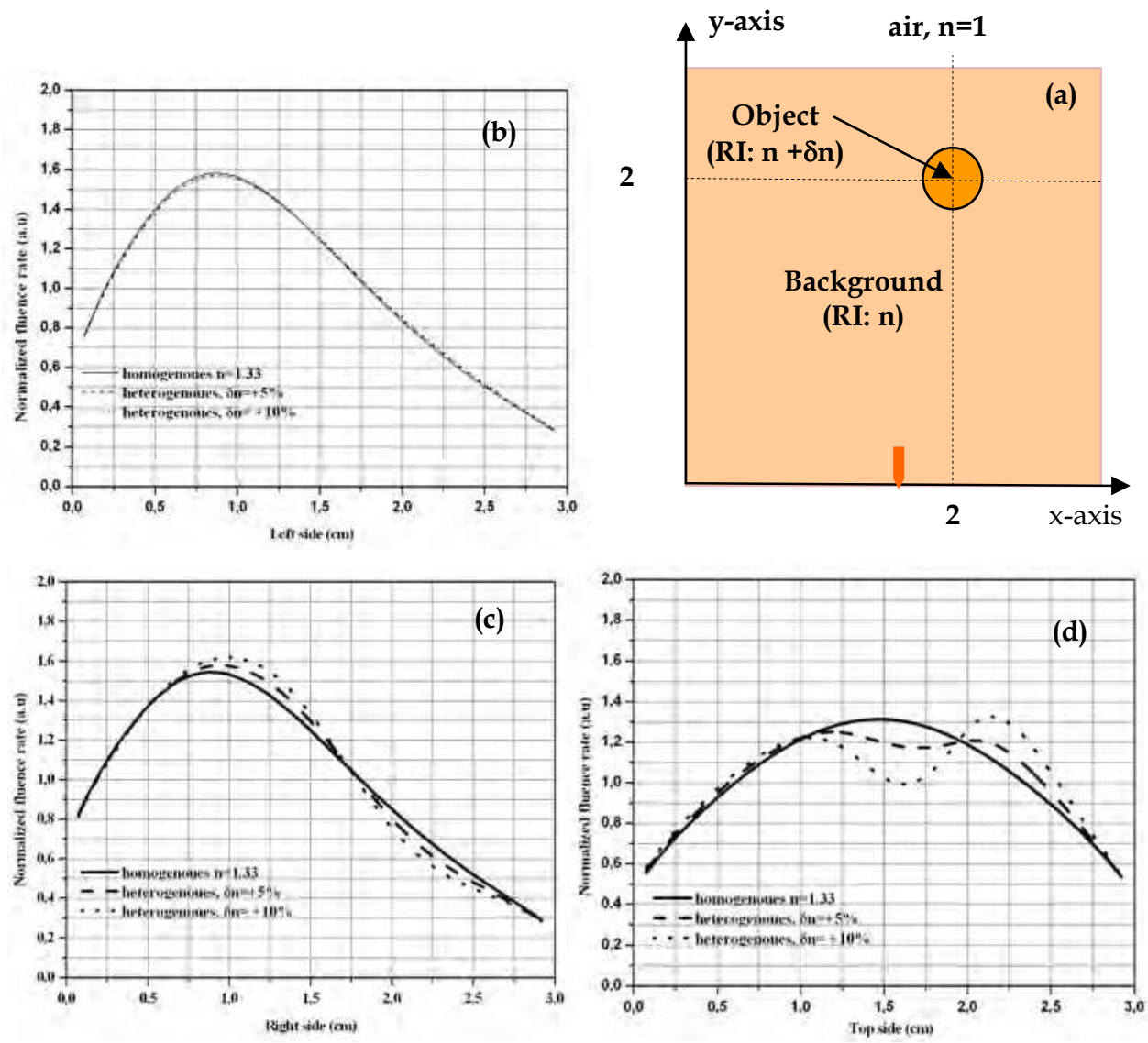

Fig. 3. Detection of heterogeneous objects by the effect of refractive index variation. (a) Geometry of the considered medium and object, (b) Detected fluence rate on the left side,(c) Detected fluence rate on the right side (d) Detected fluence rate on the top side. 


\subsection{Third investigation: Detection of inclusions in a biological tissue}

In the course of this investigation, we simulate a post-mortem human prostate tissue-like medium containing two inclusions. The geometry of the medium and the targets of the inclusions are shown in figure 3 . Each inclusion is a circle of area $0.5 \mathrm{~cm}^{2}$. The background medium properties at $850 \mathrm{~nm}$ wavelength are taken from [30, 31]. Inclusion 1 is double scattering only and inclusion 2 is double absorbing only. The refractive index of the two inclusions is taken the same and it is noted $n_{2}$. Detected fluence rate on the top and on the lateral sides of the medium is computed and presented in figure 5 and figure 6 . In particular, we confront results from refractive matching case $\left(\mathrm{n}_{1}=\mathrm{n}_{2}\right)$ and from refractive mismatching case $\left(n_{2}>n_{1}\right)$ on each side. According to figure 5 , the introduction of refractive index with scattering and absorbing parameters gives a distinguish effect on top side detected signal. However this effect is more attenuated in lateral sides because of the strong forward scattering within the medium. This is shown in figure 6.

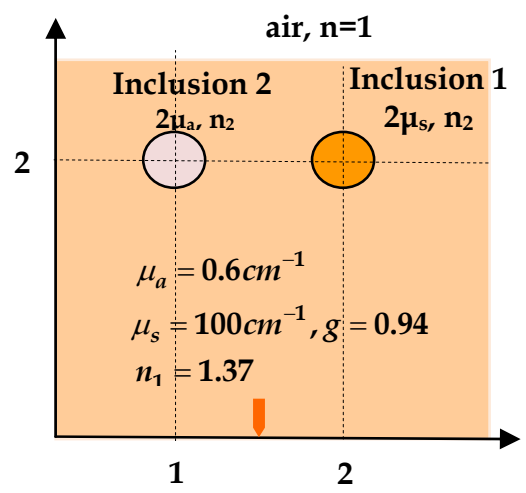

Fig. 4. Heterogeneous inclusions in human prostate-like tissue: geometry of the medium and inclusions.
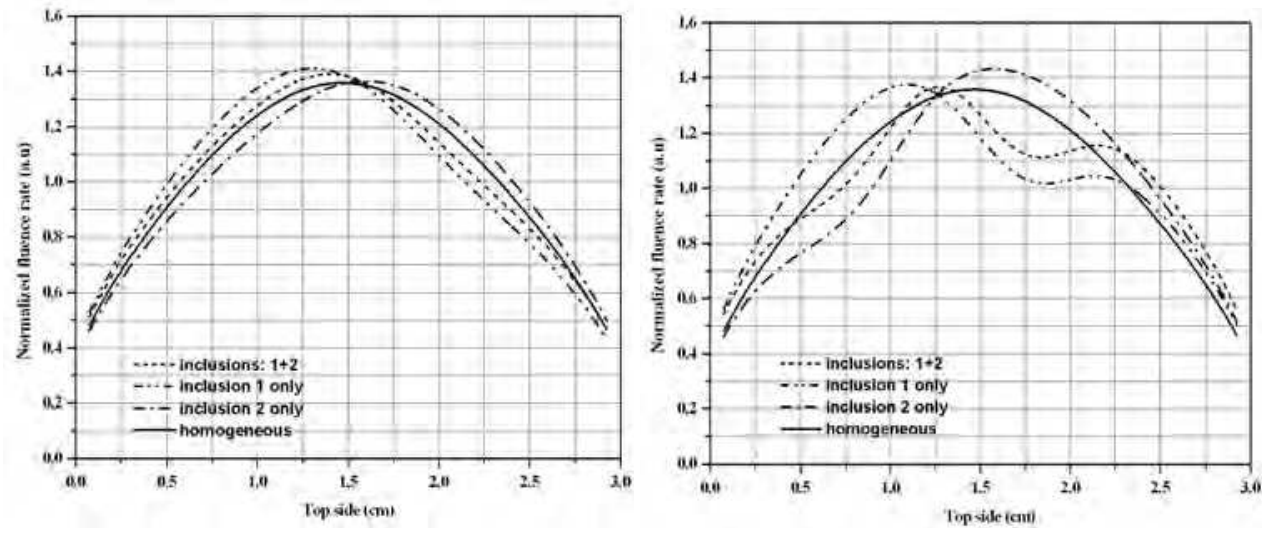

Fig. 5. Effect of heterogeneous inclusions on detected fluence rate on the top side (a) in the case of refractive matching $\left(\mathrm{n}_{1}=\mathrm{n}_{2}=1.37\right)$ and $(b)$ in the case of refractive mismatching $\left(\mathrm{n}_{1}=1.37\right.$ and $\left.\mathrm{n}_{2}=1.51\right)$. 

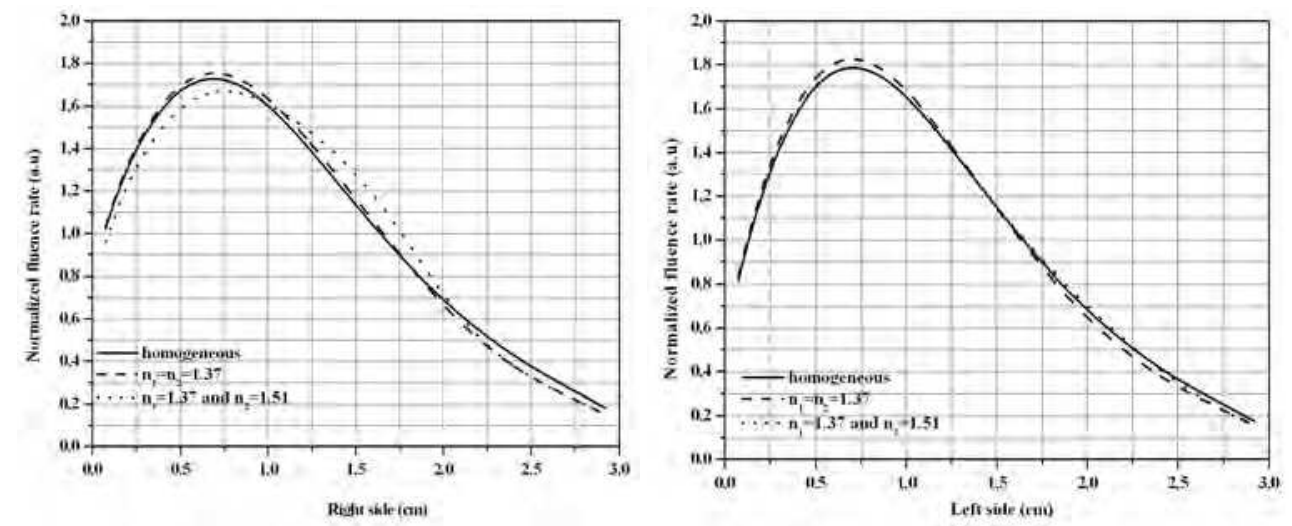

Fig. 6. Detected fluence rate on lateral sides in both cases refractive matching and mismatching cases: (a) Response on the right side (effect of inclusion 1) and (b) Response on the left side ( effect of inclusion 2)

\subsection{Fourth investigation: Effect of refractive mismatching in multilayered tissue}

As a fourth investigation, we consider a multilayered biological tissue-like medium. It contains three superposed layers of skin, fat and muscle. Figure 7 shows the considered medium. It is $3 \mathrm{~cm} \times 3 \mathrm{~cm}$-sized medium with a fixed layer of skin $(2 \mathrm{~mm})$ and with a varying fat-layer thickness of $3 \mathrm{~mm}, 6 \mathrm{~mm}$ and $9 \mathrm{~mm}$ respectively. Optical properties of different tissues at 850nm-wavelength and refractive indices of the layers are taken from [30,31]. Calculus is carried out as in the just previous section.

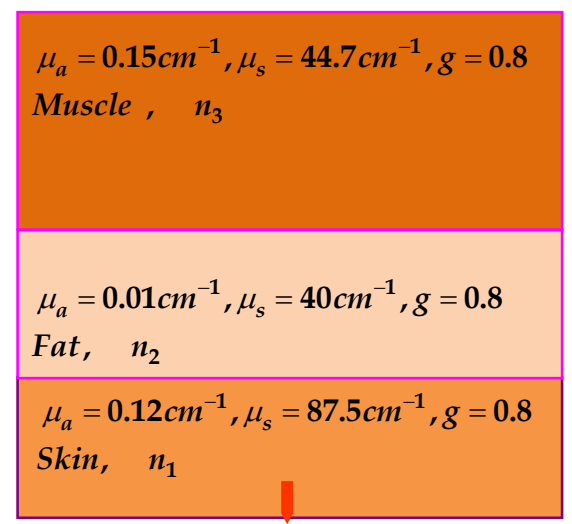

Fig. 7. A three-layered biological tissue-like medium with optical properties of skin, fat and muscle at $850 \mathrm{~nm}$-wavelength.

Figure 8 shows results concerning transmitted fluence rate on the right side of the boundary in the case of a continuous wave source. We can see that in most detector points and for the three fat-layer thicknesses transmitted fluence rate profiles are almost superposed in the case of refractive matching with evidence of detected intensity increase when the fat 
thickness increases. When taking different refractive indices, a distinct distortion in the curves at every interface is observed especially at the fat-muscle interface. In all cases much more intensity is transmitted from the muscle layer.
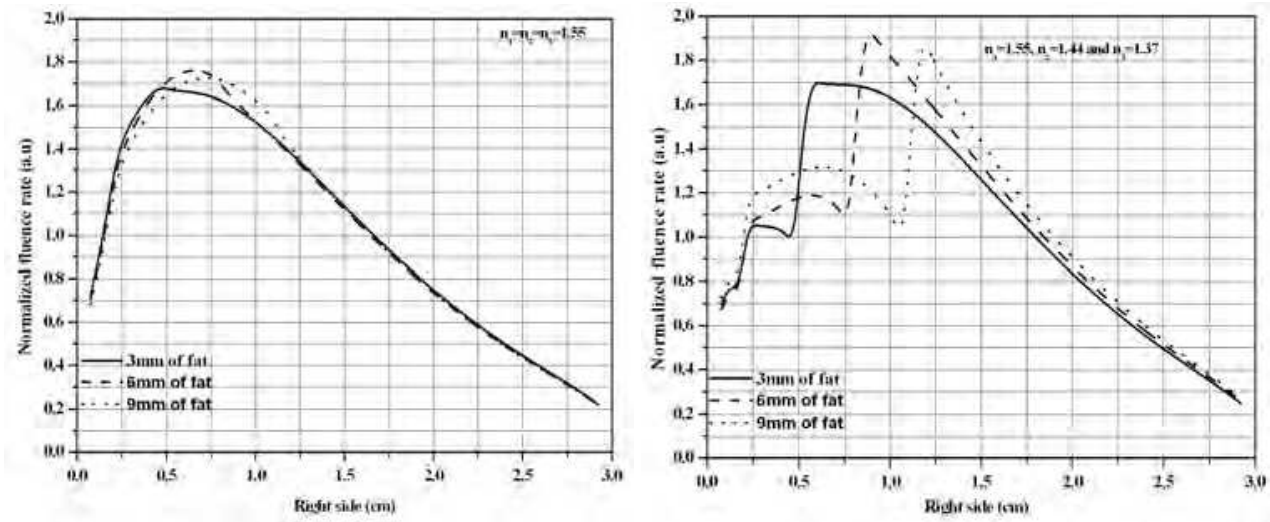

Fig. 8. Response of the three layered medium: (a) for uniform refractive index and (b) for different refractive indices.

\subsection{Fifth investigation: Detection with a short pulse}

In this investigation, we study the effect of abnormalities laying in a homogenous background medium as in the just precedent paragraph but we use a short-pulsed source. It is a 100fs-laser pulse injected in the medium through a point source on the middle of the bottom side of the boundary. Figure 9 shows the considered medium and the geometry and the properties of the
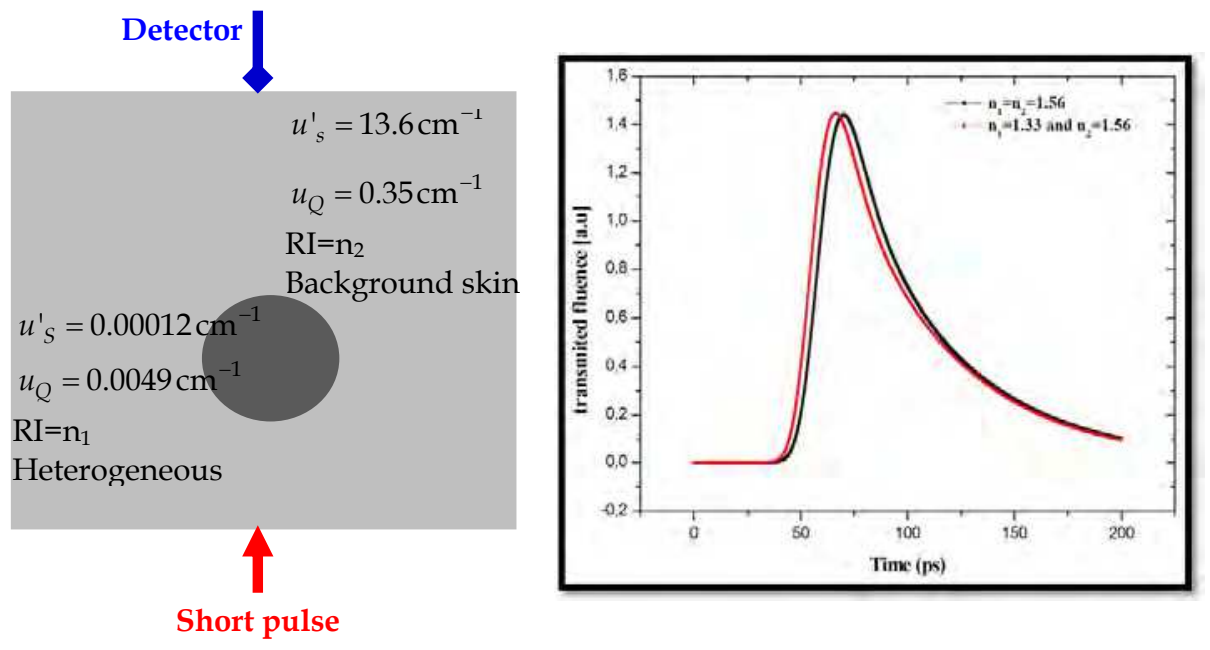

Fig. 9. A skin-like tissue containing a heterogeneous cyst-like inclusion submitted to short pulse and the detected signal. 
a circular cyst-like object laying just forward the source. The detected signal on the opposite side of the source is shown in same figure. There is a distinct phase shift due to refractive mismatching showing the presence of the cyst within the background tissue.

\subsection{Sixth investigation: Spectroscopy on a rat-liver}

In this investigation, we consider a rat liver tissue-like medium as it is shown in figure 10. Optical properties of the tissue are taken from reference [30] from a set of several wavelengths. The response illustrate transmission through the considered medium on the top side. Only near infrared light displays a relative appreciable transmission. A maximum of detected fluence rate is noted at $800 \mathrm{~nm}$-light. A little delay of the maximum detection of radiation is observed when wavelength increases. Figure 11 shows fluence rate distribution into the medium at different moments after the pulse. In all moments, symmetric propagation of light is observed into the medium. Also, it can be observed that scattering prevails in 488nm while more and more absorption is observed in $2100 \mathrm{~nm}$. At $488 \mathrm{~nm}$, the phenomenon of multiple scattering is dominant into the medium. The dispersion of the pulse is accentuated in all sides. Light disappears after 250ps. At 2100nm, more light is absorbed into the medium so a fraction of energy persists into the medium for more time.

Figure 12 shows typical movement of different photons in the medium at three different wavelengths. Calculations in our studied medium are carried out by using Monte Carlo simulations as it is described in reference. It can be observed that a typical 800nm-photon is almost snake. For $488 \mathrm{~nm}$-photon, the mean scattering free path is very small. Multiple scattering is dominant. The typical trajectory in this case is not ballistic and the photons are almost diffusive. For $2100 \mathrm{~nm}$, the mean scattering free path is longer than the $488 \mathrm{~nm}$-photon but the absorption mean free path is narrower. A typical photon in this wavelength is almost absorbed into the medium.
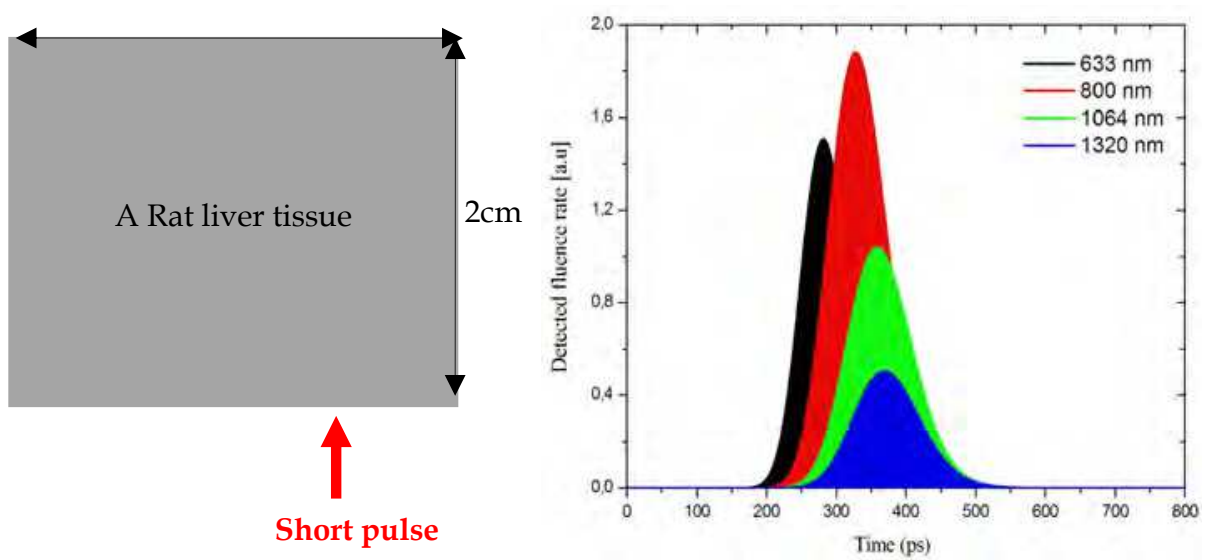

Fig. 10. Geometry of rat-liver tissue and detected signal for different wavelengths. 


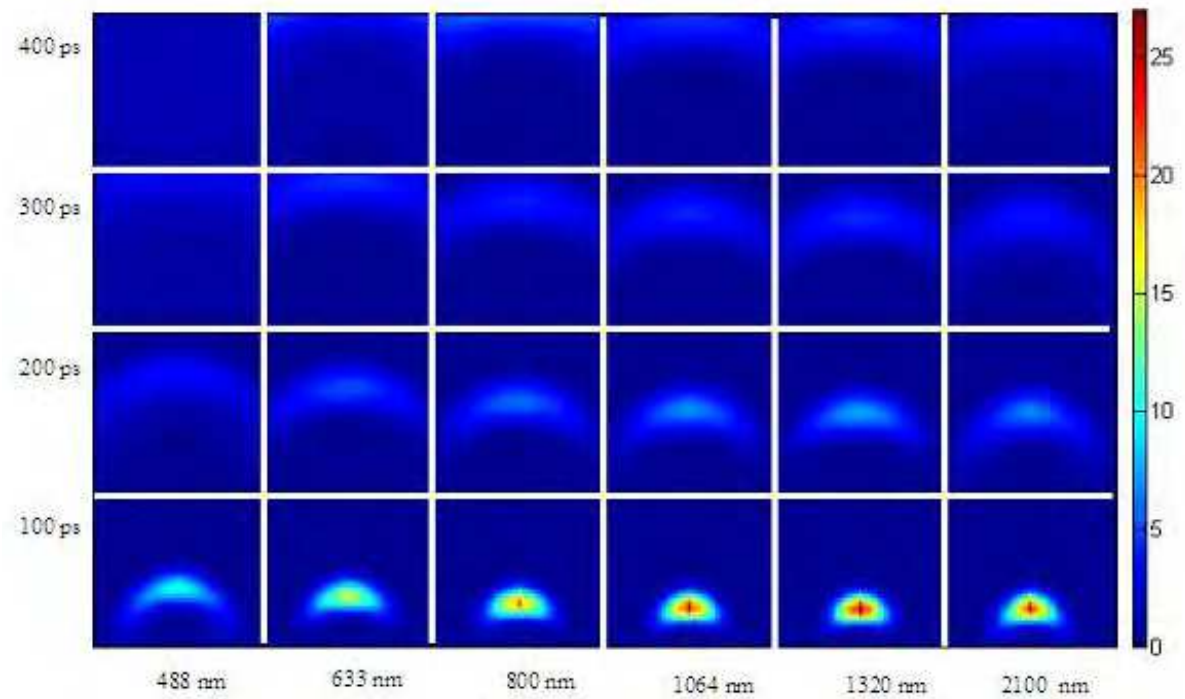

Fig. 11. Internal light distribution in the rat-liver tissue-like medium for different instants after the pulse.

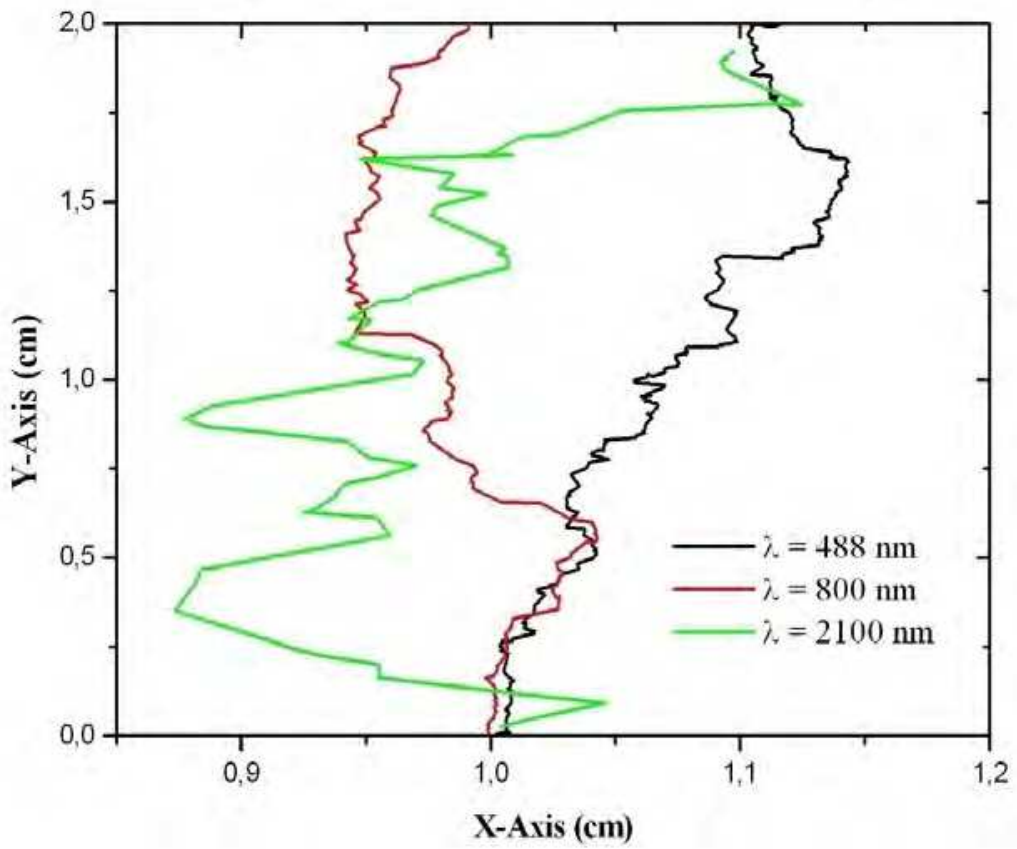

Fig. 12. Typical trajectory of photons for different values of the wavelength-source as obtained through Monte Carlo simulations. 


\section{Conclusion}

In this chapter, we attempted to develop a computational way helping in detection of abnormalities in a biological tissue. This should enable predictions of eventual tumor existence when using a diffuse optical tomography scheme. The used model is based on radiative transfer theory including a possible variation of refractive index. The model is implemented to investigate some practical situations in DOT setting and near infrared spectroscopy. Obtained results showed that variation of refractive index can yield useful predictions about the target and the location of abnormal inclusions within the tissue.

\section{Nomenclature}

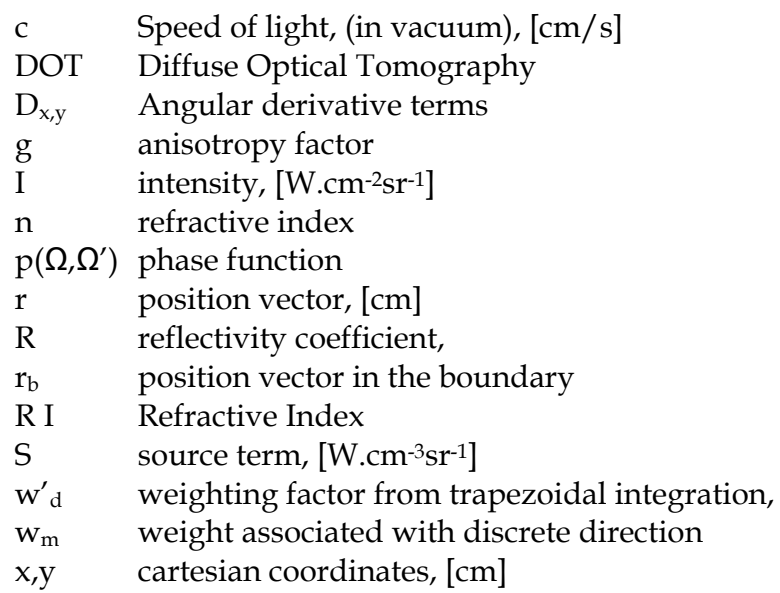

\section{Greek symbols}

$\begin{array}{ll}a & \text { interpolation parameter } \\ \Phi & \text { fluence rate, [W.cm-2] } \\ \Phi_{\mathrm{d}} & \text { Detected fluence rate, }\left[\mathrm{W} \cdot \mathrm{cm}^{-2}\right] \\ \lambda & \text { wavelength, [nm] } \\ \Omega & \text { direction solid angle, }[\mathrm{sr}] \\ \mu_{\mathrm{a}} & \text { absorption coefficient, }\left[\mathrm{cm}^{-1}\right] \\ \mu_{\mathrm{s}} & \text { scattering coefficient, }\left[\mathrm{cm}^{-1}\right] \\ \mu_{\mathrm{s}}^{\prime} & \text { reduced scattering coefficient, }\left[\mathrm{cm}^{-1}\right] \\ \rho & \text { relaxation parameter, }[-] \\ \theta & \text { scattering angle , }[\mathrm{rad}] \\ \xi, \mathrm{n} & \text { direction cosines, }[-]\end{array}$

\section{References}

[1] Selser J C, Yeh Y, Baskin R J. A light scattering characterization of membrane vesicles. Biophysical Journal; 16 (4): 337-356, (1976)

[2] Jacques S L, Prahl S A. Modeling optical and thermal distributions in tissue during laser irradiation. Lasers in Surgery and Medicine; 6: 494-503, (1987) 
[3] Arridge S R, Hebden J C. Optical imaging in medicine: II. Modeling and reconstruction. Phys Med Biol; 42: 841-853, (1997)

[4] Arridge S R, Schweiger M. A gradient-based optimisation scheme for optical tomography. Opt Express; 2 (6): 213-226, (1998)

[5] Hielscher A H, Bluestone AY, Abdoulaev AY, Klose A D, Lasker M, Stewart M, Netz U, Beuthan J. Near-infrared diffuse optical tomography. Disease Markers; 18: 313-337, (2002)

[6] Mourant J R, Hielscher A H, Eick A A, Johnson T M, Freyer J P. Evidence of intrinsic differences in the light scattering properties of tumoregenic and nontumorigenic cells. Cancer (Cancer Cytopathology); 84 (6): 366-384, (1998)

[7] Ntziachristos V, Hielscher A H, Yodh A G, Chance B. Diffuse Optical Tomography of Highly Heterogeneous Media. IEEE transactions on medical imaging; 20(6) :470$478,(2001)$

[8] Pu Y, Wang W.B , Das BB, Alfano RR.Time-resolved spectral wing emission kinetics and optical imaging of human cancerous and normal prostate tissues. Optics communications; 282:4308-4314, (2009)

[9] Klose, A, D, Netz,U, Beuthan,J and Hielscher, A, H, Optical tomography using the timeindependent equation of radiative transfer. Part I: Forward model, JQSRT, 72: 715736, (2002)

[10] Cai W, Xiaohui Ni, Gayen S K, and Alfano R R. Analytical cumulant solution of the vector radiative transfer equation investigates backscattering of circularly polarized light from turbid media. Phys. Rev. E (74) 056605, (2006)

[11] Gantri M, Trabelsi H, Bensalah R and Sediki E. Solution of a radiative transfer problem in a biological tissue. An optical tomography model. AIP Conf. Proc; 935: 237-243, (2007)

[12] Trabelsi H, Gantri M and Sediki E. Visible and Near Infrared Laser Radiation in a Biological Tissue. A Forward Model for Medical Imaging by Optical Tomography. Lasers in Med Sci; 25:41-53, (2010)

[13] Gantri M, Trabelsi H, Sediki E, Ben Salah R. Computational Laser Spectroscopy in a Biological tissue. Journal of Biophysics, article ID 253763, 9pages, (2010)

[14] Li H, Xie S. Measurement method of the refractive index of biotissue by total internal reflection. Applied Optics; 35(10):1793-1795, (1996)

[15] Das B B, Liu F , Alfano R R. Time-resolved fluorescence and photon migration studies in biomedical and model random media. Rep. Prog. Phys; 60: 227-292, (1997)

[16] Ohmi M, Ohnishi Y, Yoden K, Haruan H. In vitro simultaneous measurement of refractive index and thickness of biological tissue by the low coherence interferometry. IEEE Transactions on Biomedical Engineering; 47(9):1266-1270, (2000)

[17] Lai J, Li Z, Wang C, He A. Experimental measurement of the refractive index of biological tissues by total internal reflection. Applied Optics; 44(10):1845-1849, (2005)

[18] Beuthan J, Minet O, Helfmann J, Herrig M, Muller G. The spatial variation of the refractive index of biological cells. Phys Med Biol; 41:369-82 (1996)

[19] Dehghani H, Brooksby B, Vishwanath, K, Pogue B W, Paulsen K D. The effects of internal refractive index variation in near-infrared optical tomography: a finite element modelling approach, Physics in Medicine and Biology; 48 (16):2713-2727, (2003) 
[20] Khan T, Jiang H. A new diffusion approximation to the radiative transfer equation for scattering media with spatially varying refractive indices. J. Opt. A: Pure App. Opt; 5:137-141 (2003)

[21] Shendeleva M L, Molly, J, A, Scaling property of the diffusion equation for light in a turbid medium with varying refractive index, Journal of the Optical Society of America A; 24 (9): 2902-2910, (2007)

[22] Trabelsi H, Gantri M, Sediki E, Ben Salah R. A Time-dependent Computational Model for Radiation in a Spatially Varying Refractive Index Biological Medium. Proceedings of Eurotherm 83, Computational Thermal Radiation in Participating Media III; Lisbon, Portugal, (2009)

[23] Lemonnier D, LeDez V. Discrete ordinate solution of radiative transfer across a slab with variable refractive index. JQSRT; 72(2/5): 195-204, (2005)

[24] Liu, L, H, Benchmark numerical solutions for radiative heat transfer in two-dimensional medium with graded index distribution, JQSRT; 102: 293 - 303, (2006)

[25] Bal G. Radiative transfer equations with varying refractive index: a mathematical perspective, Journal of the Optical Society of America A; 23: 1639-1644 (2006)

[26] Ferwerda, H, A, Radiative transfer equation for scattering media with a spatially varying refractive index, Journal of Optics A: Pure and Applied Optics; 1(3): L1-L2, (1999)

[27] Tualle J M, Tinet E. Derivation of the radiative transfer equation for scattering media with a spatially varying refractive index, Optics communications; 228:33-38, (2003)

[28] Modest M, F. Radiative heat transfer; ISBN 0-12-503-163-7, Academic Press, (2003)

[29] Trabelsi H , Sghaier T et Sifaoui M S. Theoretical Study of Radiation between two Concentric Spheres Using a Modified Discrete Ordinates Method associated with Legendre Transform, JQRST; 93:415-428, (2005)

[30] Cheong W F, Prahl S A, Welch A J. A Review of the Optical Properties of Biological Tissues. IEEE J. Quantum Electronics; 26:2166-2185, (1990)

[31] Mobley J and Vo-Dinh T. optical properties of tissues, biomedical photonics handbook; ISBN 0-8493-1116-0, CRC Press LLC (2003) 


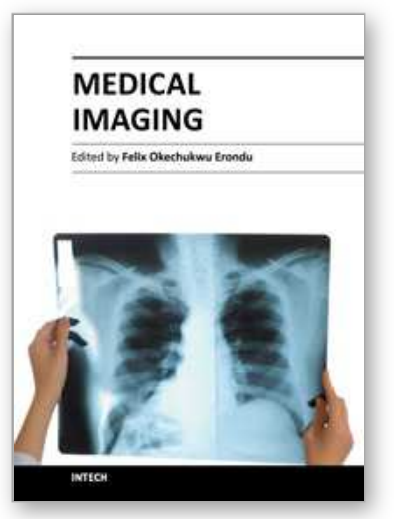

\author{
Medical Imaging \\ Edited by Dr. Okechukwu Felix Erondu
}

ISBN 978-953-307-774-1

Hard cover, 412 pages

Publisher InTech

Published online 22, December, 2011

Published in print edition December, 2011

What we know about and do with medical imaging has changed rapidly during the past decade, beginning with the basics, following with the breakthroughs, and moving on to the abstract. This book demonstrates the wider horizon that has become the mainstay of medical imaging sciences; capturing the concept of medical diagnosis, digital information management and research. It is an invaluable tool for radiologists and imaging specialists, physicists and researchers interested in various aspects of imaging.

\title{
How to reference
}

In order to correctly reference this scholarly work, feel free to copy and paste the following:

H. Trabelsi, M. Gantri and E. Sediki (2011). Detection of Abnormalities in a Biological Tissue by Diffuse Optical Tomography: A Computational Study, Medical Imaging, Dr. Okechukwu Felix Erondu (Ed.), ISBN: 978-953307-774-1, InTech, Available from: http://www.intechopen.com/books/medical-imaging/detection-ofabnormalities-in-a-biological-tissue-by-diffuse-optical-tomography-a-computational-stud

\section{INTECH}

open science | open minds

\section{InTech Europe}

University Campus STeP Ri

Slavka Krautzeka 83/A

51000 Rijeka, Croatia

Phone: +385 (51) 770447

Fax: +385 (51) 686166

www.intechopen.com

\section{InTech China}

Unit 405, Office Block, Hotel Equatorial Shanghai

No.65, Yan An Road (West), Shanghai, 200040, China

中国上海市延安西路65号上海国际贵都大饭店办公楼 405 单元

Phone: +86-21-62489820

Fax: $+86-21-62489821$ 
(C) 2011 The Author(s). Licensee IntechOpen. This is an open access article distributed under the terms of the Creative Commons Attribution 3.0 License, which permits unrestricted use, distribution, and reproduction in any medium, provided the original work is properly cited. 